\title{
Th-1 and Th-2 cytokine production in infants with virus-associated wheezing
}

\author{
P.M.C. Pitrez ${ }^{1}$, \\ D.C. Machado ${ }^{2}$, \\ M.H. Jones ${ }^{1}$, F. Andrade ${ }^{1}$, \\ C. Camozzato ${ }^{1}$ and \\ R.T. Stein ${ }^{1}$
}

\author{
${ }^{1}$ Laboratório de Pediatria e Neonatologia, Departamento de Pediatria, and \\ ${ }^{2}$ Laboratório de Pneumologia, Departamento de Medicina Interna, \\ Instituto de Pesquisas Biomédicas, Pontifícia Universidade Católica do Rio Grande \\ do Sul, Porto Alegre, RS, Brasil
}

\begin{abstract}
Correspondence
P.M.C. Pitrez

Rua Cel. Corte Real, 386/801

90630-080 Porto Alegre, RS

Brasil

Fax: +55-51-3384-5104

E-mail: pmpitrez@pucrs.br

Research supported by FAPERGS and PUCRS, Brazil.
\end{abstract}

$\ldots \ldots \ldots \ldots \ldots \ldots \ldots$

Received January 5, 2004 Accepted November 22, 2004

\begin{abstract}
Wheezing associated with respiratory viral infections in infancy is very common and results in high morbidity worldwide. The Th1/Th2 pattern of immune response in these patients remains unclear and previous studies have shown controversial results. The aim of the present study was to compare the type of Th1/Th2 cytokine response between infants with acute bronchiolitis, recurrent wheezing and upper respiratory infections from a developing country. Infants younger than 2 years of age admitted to Hospital São Lucas, Porto Alegre, RS, Brazil, between May and November 2001, with an acute episode of wheezing associated with viral respiratory infection were selected. Subjects with upper respiratory infections from the emergency department were selected for the control group. Interferon- $\gamma($ IFN- $\gamma)$ and interleukin-4 (IL-4) levels from nasal aspirates were determined by ELISA from peripheral mononuclear cell cultures. Twenty-nine subjects with acute bronchiolitis, 18 with recurrent wheezing and 15 with upper respiratory infections were enrolled. There were no differences in family history of atopy or parental smoking between groups. Oxygen requirement was similar for the acute bronchiolitis and recurrent wheezing groups. The percentage of positive tests for the cytokines studied and the IFN- $\gamma /$ IL- 4 ratio was similar for all groups. Comparison of the polarized Th1/Th2 cytokine results for the various groups showed no specific pattern of cytokine production. Infants with wheezing from a developing country do not show any specific predominant pattern of Th1/Th2 cytokine production, suggesting that multiple factors may be involved in the pathogenesis of this illness.
\end{abstract}

Key words

- Acute bronchiolitis

- Inflammation

- Interferon- $\gamma$

- Interleukin-4

- Cytokines
Wheezing in infancy is very common worldwide. Inflammation of the airways has been widely studied as a main feature involved in the pathogenesis of wheezing associated with respiratory viral infections in early life (1). However, the pattern of lymphocytic cytokine response to viral insults occurring in wheezing infants is not clear. Th1 lymphocyte cytokine production (interferon- $\gamma$, IFN- $\gamma$, and interleukin-2, IL-2) is responsible for the cell-mediated immune response. Th2 lymphocyte cytokine production (IL-4 and IL-5) stimulates antibody production, especially IgE response, also en- 
hancing eosinophil production and activity $(2,3)$. Previous reports have shown contradictory results regarding acute bronchiolitis (AB), indicating that either Th1 or Th2 responses may predominate in this disease (46). Other studies, however, have reported that there is no specific Th1 or Th2 pattern of response in infants with $\mathrm{AB}(7,8)$.

Recent data comparing asthma prevalence between developed and non-developed countries have shown that poorer populations present a lower asthma/wheezing prevalence than more affluent societies $(9,10)$. Viral, bacterial and parasitic infections are some of the associated risk factors that can influence the different frequencies of asthma between these populations (11). To our knowledge, there are no previous studies of the Th1/Th2 response during acute wheezing episodes in infants from a developing country population.

Thus, the aim of the present study was to determine whether an imbalance of either Th1 or Th2 predominates in infants from a developing country population with viralassociated acute wheezing.

Infants admitted to Hospital São Lucas, Pontifícia Universidade Católica do Rio Grande do Sul, Porto Alegre, RS, Brazil, between May and September 2001, with acute wheezing and symptoms of viral respiratory infection including clear or mucoid rhinorrhea, cough and tachypnea, were selected for the study. Family history of asthma, parental smoking and oxygen requirement were recorded. Subjects with previous cardiopulmonary diseases or steroid treatment were excluded. Subjects were then classified as having the first episode of wheezing (AB) or recurrent wheezing (RW). Infants with upper respiratory infection (URI) were selected as the control group from the emergency department. URI subjects had no wheezing or crackles on examination and had oxygen saturation $>96 \%$.

The study was approved by the Ethics Committee of Hospital São Lucas. All par- ents gave written informed consent.

Samples of nasopharyngeal aspirate (NPA) were obtained within the first $48 \mathrm{~h}$ of admission by placing a polyethylene catheter in the nasopharynx and applying gentle suction into a mucous trap. The catheter was flushed with $1 \mathrm{ml}$ phosphate-buffered saline.

The samples were then centrifuged at $2000 \mathrm{rpm}$ for $2 \mathrm{~min}$ and the supernatant was removed and frozen $\left(-80^{\circ} \mathrm{C}\right)$ for cytokine analysis. NPA was stained by immunofluorescence for respiratory syncytial virus (RSV).

The concentrations of IFN- $\gamma$ and IL-4 were determined by ELISA (Pharmingen, San Diego, CA, USA), with a detection limit of 4.7 and $7.8 \mathrm{pg} / \mathrm{ml}$, respectively. Results were considered to be negative when cytokine concentrations were below the detection limit. Polarized (Th1 or Th2) cytokine results were considered to be present when one cytokine was detected and the other was negative.

Cytokine level results are presented as median and interquartile. The Mann-Whitney test and ANOVA were used to compare cytokine levels between the groups studied, with the level of significance set at $\mathrm{P}<0.05$.

Twenty-nine subjects with $\mathrm{AB}, 18$ with RW and 15 with URI were enrolled in the study. The characteristics of the subjects studied are shown in Table 1. Infants with $\mathrm{AB}$ were younger than the other groups studied $(\mathrm{P}<0.001)$. There was no difference in family history of asthma or parental smoking between the groups studied. Oxygen requirement was similar for the subjects with $\mathrm{AB}$ and RW. RSV infection was detected in 22 (76\%) subjects with $\mathrm{AB}, 10$ (56\%) subjects with RW and 7 (47\%) subjects with URI. The prevalence of RSV infection was not significantly different between the groups studied. None of the AB and RW subjects studied required mechanical ventilation.

The percentage of positive tests for the cytokines studied and the IFN- $\gamma /$ IL- 4 ratio was similar for the groups studied. The me- 
dian levels of cytokines studied also did not differ significantly between groups (Table 1).

Neither IFN- $\gamma$ nor IL-4 were detected in 21 subjects with $A B$ and 10 with RW. However, skewed cytokine production and a mixed pattern were detected in both groups. When values of IFN- $\gamma$ levels in NPA were plotted against IL-4 levels and the polarized Th1/Th2 cytokine results were analyzed in these subjects, no specific pattern of the type of cytokines produced was detected in subjects with $\mathrm{AB}$ and RW (Figure 1).

It was not possible to compare the severity of $\mathrm{AB}$ episodes between polarized and non-polarized subjects because of the small sample size. There was no difference in immune response between the groups studied when data were analyzed separately by virus type.

In the present study, virus-associated wheezing in infancy was not associated with any specific pattern of lymphocyte cytokine production. It is not clear whether a Th1 or Th2 response may be triggered by the respiratory viruses themselves or may be particularly regulated by the intrinsic host immune response pattern.

In the late 1990's, many studies focused on the pattern of lymphocyte cytokine production in $\mathrm{AB}$. Many reports have shown that $\mathrm{AB}$ due to RSV may be associated with a Th2-lymphocyte response $(4,5,12,13)$. IL4 production during $\mathrm{AB}$ could be induced by RSV infection and could be associated with the development of lower airway tract disease in infancy. Moreover, Renzi and colleagues (5) have found an association of increased IL-4 with wheezing after AB in response to Dermatophagoides farinae. However, other studies have shown contradictory results. An increased IFN- $\gamma$ production was found in infants with $\mathrm{AB}$ and recurrent wheezing $(6,14)$.

In the present study, we have shown that the development of $\mathrm{AB}$ and $\mathrm{RW}$ in infancy is not related to a uniformly skewed pattern of cytokine lymphocyte production. Our results agree with recent data showing both Th1 and Th2 cytokine release in infants with lower respiratory tract illness caused by RSV (7). Moreover, Mobbs and colleagues (8) have also shown that divergent $\mathrm{T}$-cell responses can be found in severe RSV-induced AB. In

Table 1. Characteristics of the subjects studied and detection of IFN- $\gamma$ and IL-4 levels in nasopharyngeal aspirate.

\begin{tabular}{lcccc}
\hline & $A B(N=29)$ & $R W(N=18)$ & URI (N =15) & $P$ \\
\hline $\begin{array}{l}\text { Median age in months } \\
\text { (interquartile) }\end{array}$ & 3.5 & 6.8 & 8.4 & $<0.001$ \\
Male sex, \% & $(2.6-6.3)$ & $(5.3-12.2)$ & $(6.8-15.6)$ & \\
Parental smoking, \% & 24 & 14 & 10 & NS \\
Family history of atopy, \% & 20 & 17 & 9 & NS \\
RSV detected, \% & 15 & 15 & 9 & NS \\
Oxygen requirement, \% & 76 & 56 & 47 & NS \\
IFN- $\gamma(\%)$ & $6(21)$ & $6(33)$ & $2(13)$ & NS \\
IL-4 (\%) & $7(24)$ & $6(33)$ & $3(20)$ & NS \\
IFN- $\gamma+$ IL-4 (\%) & $5(17)$ & $5(28)$ & $1(7)$ & NS \\
\hline
\end{tabular}

$\mathrm{AB}=$ acute bronchiolitis; $\mathrm{RW}=$ recurrent wheezing; $\mathrm{URI}=$ upper respiratory infection; $\mathrm{RSV}=$ respiratory syncytial virus; IFN- $\gamma=$ interferon $\gamma$; IL-4 = interleukin 4 ; $\mathrm{NS}=$ nonsignificant.

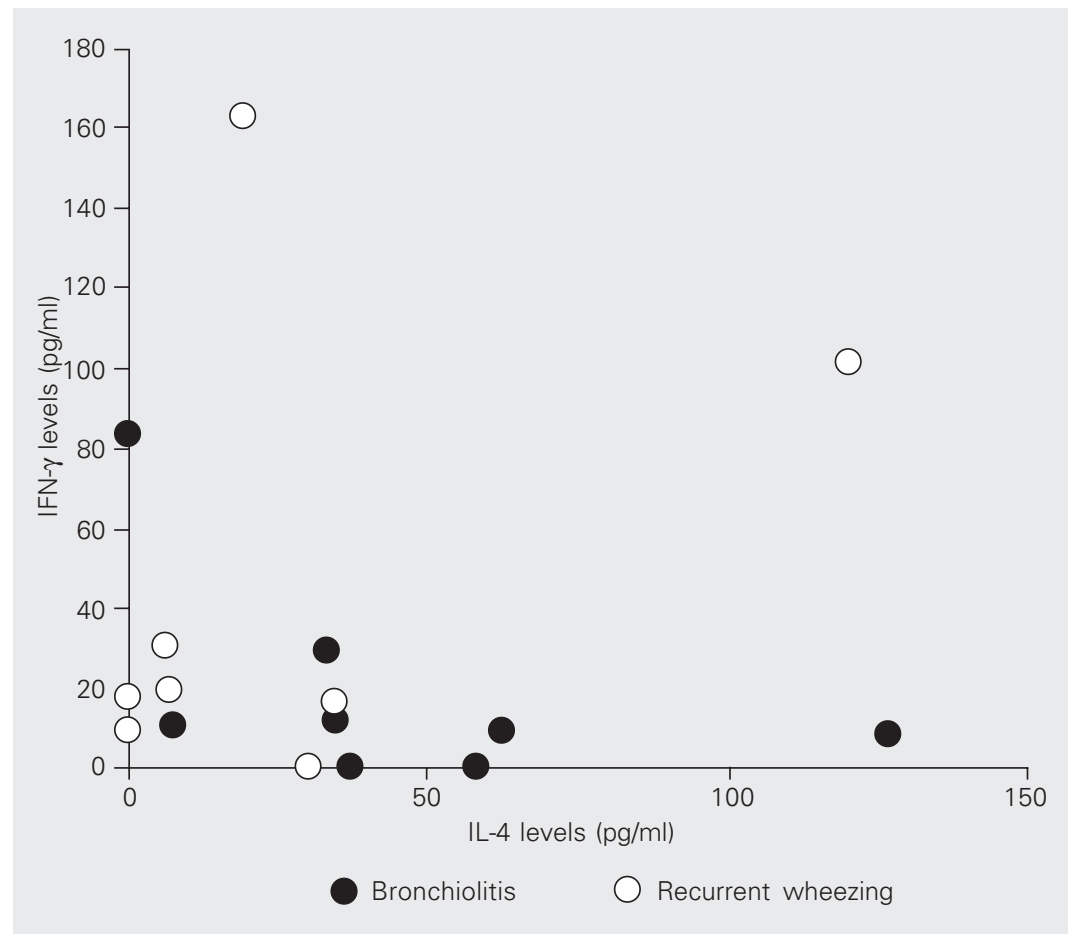

Figure 1. Relationship between IFN- $\gamma$ and IL-4 levels in nasopharyngeal aspirate from infants with acute bronchiolitis and recurrent wheezing. 
our study, some individuals produced either a skewed or mixed pattern of cytokine production and others had undetectable levels of IFN- $\gamma$ or IL-4 in NPA. These findings suggest that the type of lymphocyte response may be individually related to the genetic background of the host immune response or to environmental factors. This issue could not be considered in our study because we did not analyze environmental factors and our sample was not large enough for this type of analysis.

Given that our population sample was selected from a developing country, we could hypothesize that many environmental factors (parasitic/bacterial infections and endotoxin exposure) may influence the development of the immune response during infancy. However, the group of subjects studied was very young and environmental factors would not have a significant impact in this early stage of life. Moreover, our findings also suggest that the virus-induced im- mune response is not responsible for a uniform pattern of lymphocyte cytokine release.

The present results suggest that the pattern of lymphocyte production after acute bronchiolitis or recurrent wheezing is not uniform. The "genetic" characteristic of the Th1/Th2 response and environmental factors may influence the immune response established during and after AB. Further studies with larger samples and a longitudinal design with subjects from different socioeconomic levels in different countries are required to elucidate the role of the Th1 and Th2 response associated with the development of $\mathrm{AB}$ and wheezing in infancy.

\section{Acknowledgments}

The authors wish to thank the nurses, pediatricians and the immunology laboratory staff of Hospital São Lucas, PUCRS, for their help and cooperation.

\section{References}

1. van Schaik SM, Welliver RC \& Kimpen JLL (2000). Novel pathways in the pathogenesis of respiratory syncytial virus disease. Pediatric Pulmonology, 30: 131-138.

2. Chung KF \& Barnes PJ (1999). Cytokines in asthma. Thorax, 54: 825-857.

3. Mosmann TR \& Sad S (1996). The expanding universe of T-cell subsets: Th1, Th2 and more. Immunology Today, 17: 138-146.

4. Román M, Calhoun WJ, Hinton KL, Avendaño LF, Simon V, Escobar AM, Gaggero A \& Díaz PV (1997). Respiratory syncytial virus infection in infants is associated with predominant Th-2-like response. American Journal of Respiratory and Critical Care Medicine, 156: 190-195.

5. Renzi PM, Turgeon JP, Yang JP, Drblik SP, Marcotte JE, Pedneault L \& Spier S (1997). Cellular immunity is activated and a TH-2 response is associated with early wheezing in infants after bronchiolitis. Journal of Pediatrics, 130: 584-593.

6. van Shaik SM, Tristam DA, Nagpal IS, Hintz KM, Welliver II RC \& Welliver RC (1999). Increased production of INF- $\gamma$ and cysteinyl leukotrienes in virus-induced wheezing. Journal of Allergy and Clinical Immunology, 103: 630-636.

7. Tripp RA, Moore D, Barskey IV A, Jones L, Moscatiello C, Keyserling $H$ \& Anderson LJ (2002). Peripheral blood mononuclear cells from infants hospitalized because of respiratory syncytial virus infection express $T$ helper- 1 and $T$ helper- 2 cytokines and CC chemokine messenger RNA. Journal of Infectious Diseases, 185: 1388-1394.

8. Mobbs KJ, Smyth RL, O'Hea U, Ashby D, Ritson P \& Hart A (2002).
Cytokines in severe respiratory syncytial virus bronchiolitis. Pediatric Pulmonology, 33: 49-52.

9. The International Study of Asthma and Allergies in Childhood (ISAAC) Steering Committee (1998). Worldwide variation in prevalence of symptoms of asthma, allergic rhinoconjunctivitis, and atopic eczema: ISSAC. Lancet, 351: 1225-1232.

10. Von Mutius E, Martinez FD, Fritzsch C, Nicolai T, Roell G \& Thiemann $H$ (1994). Prevalence of asthma and atopy in two areas of West and East Germany. American Journal of Respiratory and Critical Care Medicine, 149: 358-364

11. Von Mutius E (2001). Infection: friend or foe in the development of atopy and asthma? The epidemiological evidence. European Respiratory Journal, 18: 872-881.

12. Pala $P$, Bjarnason $R$, Sigurbergsson $F$, Metcalfe $C$, Sigurs N \& Openshaw PJM (2002). Enhanced IL-4 responses in children with a history of respiratory syncytial virus bronchiolitis in infancy. European Respiratory Journal, 20: 376-382.

13. Legg PJ, Hussain IR, Warner JA, Johnston SL \& Warner JO (2003). Type 1 and type 2 cytokine imbalance in acute respiratory syncytial virus bronchiolitis. American Journal of Respiratory and Critical Care Medicine, 168: 633-639.

14. Brandenburg $A H$, Kleinjan $A$, van het Land $B$, Moll HA, Timmerman HH, de Swart RI, Neijens HJ, Fokkens H \& Osterhaus ADME (2000). Type 1-like immune response is found in children with respiratory syncytial virus infection regardless of clinical severity. Journal of Medical Virology, 62: 267-277. 